\title{
LONGITUDINAL KAM-COCYCLES AND ACTION SPECTRA OF MAGNETIC FLOWS
}

\author{
Nurlan S. Dairbekov and Gabriel P. Paternain
}

\begin{abstract}
Let $M$ be a closed oriented surface and let $\Omega$ be a non-exact 2 -form. Suppose that the magnetic flow $\phi$ of the pair $(g, \Omega)$ is Anosov. We show that the longitudinal KAM-cocycle of $\phi$ is a coboundary if and only if the Gaussian curvature is constant and $\Omega$ is a constant multiple of the area form thus extending the results in [12]. We also show infinitesimal rigidity of the action spectrum of $\phi$ with respect to variations of $\Omega$. Both results are obtained by showing that if $G: M \rightarrow \mathbb{R}$ is any smooth function and $\omega$ is any smooth 1-form on $M$ such that $G(x)+\omega_{x}(v)$ integrates to zero along any closed orbit of $\phi$, then $G$ must be identically zero and $\omega$ must be exact.
\end{abstract}

\section{Introduction}

Let $M$ be a closed oriented surface endowed with a Riemannian metric $g$ and let $\Omega$ be a 2 -form. The magnetic flow of the pair $(g, \Omega)$ is the flow $\phi$ on the unit sphere bundle $S M$ determined by the equation

$$
\frac{D \dot{\gamma}}{d t}=\lambda(\gamma) i \dot{\gamma}
$$

where $i$ indicates rotation by $\pi / 2$ according to the orientation of the surface and $\lambda$ is the smooth function on $M$ uniquely determined by $\Omega=\lambda \Omega_{a}$, where $\Omega_{a}$ is the area form of $M$. When $\Omega$ vanishes we recover the usual geodesic flow of the surface. A curve $\gamma: \mathbb{R} \rightarrow M$ that solves (1) will be called a magnetic geodesic.

In the present paper we shall study rigidity properties of Anosov magnetic flows. The Anosov property means that $T(S M)$ splits as $T(S M)=E^{0} \oplus E^{u} \oplus E^{s}$ in such a way that there are constants $C>0$ and $0<\rho<1<\eta$ such that $E^{0}$ is spanned by the generating vector field of the flow, and for all $t>0$ we have

$$
\left\|\left.d \phi_{-t}\right|_{E^{u}}\right\| \leq C \eta^{-t} \text { and }\left\|\left.d \phi_{t}\right|_{E^{s}}\right\| \leq C \rho^{t} .
$$

The subbundles are then invariant and Hölder continuous and have smooth integral manifolds, the stable and unstable manifolds, which define a continuous foliation with smooth leaves.

To any $C^{k}$ volume preserving Anosov flow $\varphi$ on a closed 3-manifold $N, \mathrm{P}$. Foulon and B. Hasselblatt [4] associated its longitudinal KAM-cocycle. This is a cocycle that measures the regularity of the subbundle $E^{u} \oplus E^{s}$ The main theorem

Received by the editors January 12, 2005. 
in [4] asserts that $E^{u} \oplus E^{s}$ is always Zygmund-regular and that the following are equivalent:

1. $E^{u} \oplus E^{s}$ is "little Zygmund";

2. the longitudinal KAM-cocycle is a coboundary;

3. $E^{u} \oplus E^{s}$ is Lipschitz;

4. $E^{u} \oplus E^{s}$ is $C^{k-1}$;

5. $\varphi$ is a suspension or contact flow.

(A continuous function $f: U \rightarrow \mathbb{R}$ on an open set $U \subset \mathbb{R}$ is said to be Zygmund-regular if $|f(x+h)+f(x-h)-2 f(x)|=O(h)$ for all $x$ in $U$. The function is said to be "little Zygmund" if $|f(x+h)+f(x-h)-2 f(x)|=o(h)$.)

It is well known that for flows, a "choice of time" or equivalently, a choice of speed at which orbits travel gets reflected on the regularity of the corresponding strong stable and strong unstable distributions. The situation is different if we look at the weak unstable and stable bundles $E^{0} \oplus E^{u}$ and $E^{0} \oplus E^{s}$. S. Hurder and A. Katok proved [7] that the weak bundles are always differentiable with Zygmund-regular derivative and there is a cocycle obstruction to higher regularity given by the first nonlinear term in the Moser normal form (this explains why Foulon and Hasselblatt used the terminology "longitudinal KAMcocycle").

In [12], the second author showed that if $\Omega$ is non-exact, $g$ has negative Gaussian curvature $K$ and $\lambda$ is small enough in the $C^{0}$ norm, then the longitudinal KAM-cocycle of $\phi$ is a coboundary if and only if $K$ and $\lambda$ are constant. In the present paper we would like to extend this result to all Anosov magnetic flows, without restrictions on curvature or the size of $\lambda$. As shown in [1] the set of Anosov magnetic flows can certainly go well beyond small perturbations of Anosov geodesic flows.

Theorem A. Let $M$ be a closed oriented surface endowed with a Riemannian metric $g$ and let $\Omega$ be an arbitrary 2-form. Suppose that the magnetic flow $\phi$ of the pair $(g, \Omega)$ is Anosov. We have:

1. If $\Omega$ is exact, then the longitudinal KAM-cocycle of $\phi$ is a coboundary if and only if $\Omega$ vanishes identically, i.e. $\phi$ is a geodesic flow;

2. If $\Omega$ is non-exact, then the longitudinal $K A M$-cocycle of $\phi$ is a coboundary if and only if the Gaussian curvature is constant and $\Omega$ is a constant multiple of the area form.

Item (1) was proved in [11] using Aubry-Mather theory, but it was stated in a different form. The main result in [11] asserts that if $\Omega$ is exact and the Anosov splitting is of class $C^{1}$, then $\Omega$ must be zero (and this holds in any dimension). The main result of Foulon and Hasselblatt tells us that, for surfaces, the conditions of $C^{1}$ Anosov splitting and longitudinal KAM-cocycle being a coboundary are equivalent.

The proof of item (2) in [12] for negative $K$ and small $\Omega$ was based on Fourier analysis using the set up of V. Guillemin and D. Kazhdan in [5]. Our approach here is based on establishing a Pestov identity for magnetic flows similar to the 
ones in $[2,3]$ for geodesic flows. Using this identity we will prove the following result which has independent interest:

Theorem B. Let $M$ be a closed oriented surface and $\Omega$ an arbitrary smooth 2-form. Suppose the magnetic flow $\phi$ of the pair $(g, \Omega)$ is Anosov and let $X_{\phi}$ be the vector field generating $\phi$. If $G: M \rightarrow \mathbb{R}$ is any smooth function and $\omega$ is any smooth 1-form on $M$ such that there is a smooth function $\varphi: S M \rightarrow \mathbb{R}$ for which $G(x)+\omega_{x}(v)=X_{\phi}(\varphi)$, then $G$ is identically zero and $\omega$ is exact.

Note that by the smooth Livšic theorem [8] saying that $G(x)+\omega_{x}(v)=X_{\phi}(\varphi)$ is equivalent to saying that $G(x)+\omega_{x}(v)$ has zero integral over every closed magnetic geodesic. Theorem A follows from Theorem B using the same methods as in [12], so we will not repeat the proof here. Instead we will consider a second application of Theorem B.

Given any closed 2-form $\Omega$, fix a constant $c \neq 0$ such that the cohomology class of $c \Omega$ is an integral class, i.e. $[c \Omega] \in H^{2}(M, \mathbb{Z})=\mathbb{Z}$. Then there exists a principal circle bundle $\Pi: P \rightarrow M$ with Euler class $[c \Omega]$. The bundle admits a connection 1-form $\alpha$ such that $d \alpha=-2 \pi c \Pi^{*} \Omega$. Recall that the holonomy function is a map $\log \operatorname{hol}_{\alpha}: Z_{1}(M) \rightarrow \mathbb{R} / \mathbb{Z}$, where $Z_{1}(M)$ is the space of 1 cycles, such that for every 2-chain $f: \Sigma \rightarrow M$ we have

$$
\log \operatorname{hol}_{\alpha}(\partial \Sigma)=-c \int_{\Sigma} f^{*} \Omega \bmod 1 .
$$

Let $\gamma$ be a closed magnetic geodesic and let $\ell(\gamma)$ be its length. We define the action of $\gamma$ as:

$$
A(\gamma):=\ell(\gamma)+c^{-1} \log \operatorname{hol}_{\alpha}(\gamma) \bmod 1
$$

We call the set $\mathcal{S} \subset \mathbb{R} / \mathbb{Z}$ of values $A(\gamma)$ as $\gamma$ ranges over all closed magnetic geodesics, the action spectrum of the magnetic flow. If all the closed orbits of the magnetic flow $\phi$ are nondegenerate, then $\mathcal{S}$ is a countable set.

Suppose now that we vary the connection 1-form $\alpha$. Let $\alpha_{\tau}$ be a smooth 1-parameter family of connections for $\tau \in(-\varepsilon, \varepsilon)$ with $\alpha_{0}=\alpha$ Then we can write $\alpha_{\tau}-\alpha=\Pi^{*} \beta_{\tau}$, where $\beta_{\tau}$ are smooth 1 -forms on $M$. The connection $\alpha_{\tau}$ has curvature form $-2 \pi c \Omega+d \beta_{\tau}$. If we let $\Omega_{\tau}=\Omega-\frac{1}{2 \pi c} d \beta_{\tau}$ we get a magnetic flow $\phi^{\tau}$ and an action spectrum $\mathcal{S}_{\tau}$. If the magnetic flow $\phi$ is Anosov, then for $\varepsilon$ small enough $\phi^{\tau}$ is Anosov for all $\tau \in(-\varepsilon, \varepsilon)$.

Theorem C. Let $M$ be a closed oriented surface endowed with a Riemannian metric $g$ and let $\Omega$ be a 2-form. Suppose the magnetic flow of the pair $(g, \Omega)$ is Anosov. If $\mathcal{S}_{\tau}=\mathcal{S}$ for all $\tau$ sufficiently small, then the deformation is trivial, that is, $\alpha_{\tau}=\alpha+\Pi^{*} d F_{\tau}$ and $\Omega_{\tau}=\Omega$, where $F_{\tau}$ are smooth functions on $M$.

Theorem $\mathrm{C}$ and the results of V. Guillemin and A. Uribe in [6] give a version of infinitesimal spectral rigidity for magnetic flows. In order to describe this rigidity we will assume that $c=1$. This is really no restriction at all since the magnetic flows of $(g, \Omega)$ and $\left(c^{2} g, c \Omega\right)$ are the same up to a constant time change. For every positive integer $m$, let $L_{m}$ be the Hermitian line bundle with 
connection over $M$ associated with $\Pi$ via the character $e^{i \theta} \mapsto e^{i m \theta}$ of $S^{1}$. The metric on $M$, together with the connection on $L_{m}$ determine a Bochner-Laplace operator acting on sections of $L_{m}$. For each $m$, let $\left\{\nu_{m, j}: j=1,2, \ldots\right\}$ be the spectrum of this operator. If we now vary the connection 1 -form $\alpha$ as above we obtain eigenvalues $\nu_{m, j}^{\tau}$.

Corollary. Let $M$ be a closed oriented surface endowed with a Riemannian metric $g$ and let $\Omega$ be an integral 2-form. Suppose the magnetic flow of the pair $(g, \Omega)$ is Anosov. If $\nu_{m, j}^{\tau}$ is independent of $\tau$ for all $m$ and $j$ (i.e. the deformation is isospectral), then the deformation is trivial, that is, $\alpha_{\tau}=\alpha+\Pi^{*} d F_{\tau}$ and $\Omega_{\tau}=\Omega$, where $F_{\tau}$ are smooth functions on $M$.

Indeed, let us consider the periodic distribution

$$
\Upsilon(s)=\sum_{m, j} \varphi\left(\sqrt{\nu_{m, j}+m^{2}}-m \sqrt{2}\right) e^{i m s}
$$

where $\varphi$ is a Schwartz function on the real line. Theorem 6.9 in [6] asserts that the singularities of $\Upsilon$ are included in the set of all $s \in \mathbb{R}$ for which $s / 2 \pi \bmod 1 \in \mathcal{S}$. Moreover, each point of the action spectrum arises as a singularity of $\Upsilon$ for an appropriate choice of $\varphi$. The corollary is now an immediate consequence of Theorem C.

Acknowledgements: The first author would like to thank the Max-PlanckInstitut für Mathematik in Bonn for hospitality and financial support while this work was in progress.

\section{Preliminaries}

Let $M$ be a closed oriented surface, $S M$ the unit sphere bundle and $\pi: S M \rightarrow$ $M$ the canonical projection. The latter is in fact a principal $S^{1}$-fibration and we let $V$ be the infinitesimal generator of the action of $S^{1}$.

Given a unit vector $v \in T_{x} M$, we will denote by $i v$ the unique unit vector orthogonal to $v$ such that $\{v, i v\}$ is an oriented basis of $T_{x} M$. There are two basic 1-forms $\alpha$ and $\beta$ on $S M$ which are defined by the formulas:

$$
\begin{aligned}
& \alpha_{(x, v)}(\xi):=\left\langle d_{(x, v)} \pi(\xi), v\right\rangle ; \\
& \beta_{(x, v)}(\xi):=\left\langle d_{(x, v)} \pi(\xi), i v\right\rangle .
\end{aligned}
$$

The form $\alpha$ is the canonical contact form of $S M$ whose Reeb vector field is the geodesic vector field $X$. The volume form $\alpha \wedge d \alpha$ gives rise to the Liouville measure $d \mu$ of $S M$.

A basic theorem in 2-dimensional Riemannian geometry asserts that there exists a unique 1 -form $\psi$ on $S M$ (the connection form) such that $\psi(V)=1$ and

$$
\begin{aligned}
& d \alpha=\psi \wedge \beta \\
& d \beta=-\psi \wedge \alpha \\
& d \psi=-(K \circ \pi) \alpha \wedge \beta
\end{aligned}
$$


where $K$ is the Gaussian curvature of $M$. In fact, the form $\psi$ is given by

$$
\psi_{(x, v)}(\xi)=\left\langle\frac{D Z}{d t}(0), i v\right\rangle
$$

where $Z:(-\varepsilon, \varepsilon) \rightarrow S M$ is any curve with $Z(0)=(x, v)$ and $\dot{Z}(0)=\xi$ and $\frac{D Z}{d t}$ is the covariant derivative of $Z$ along the curve $\pi \circ Z$.

For later use it is convenient to introduce the vector field $H$ uniquely defined by the conditions $\beta(H)=1$ and $\alpha(H)=\psi(H)=0$. The vector fields $X, H$ and $V$ are dual to $\alpha, \beta$ and $\psi$ and as a consequence of $(2-4)$ they satisfy the commutation relations

$$
[V, X]=H, \quad[V, H]=-X, \quad[X, H]=K V .
$$

Equations (2-4) also imply that the vector fields $X, H$ and $V$ preserve the volume form $\alpha \wedge d \alpha$ and hence the Liouville measure.

\section{Proof of Theorem B}

Henceforth $(M, g)$ is a closed oriented surface and $X, H$, and $V$ are the same vector fields on $S M$ as in the previous section.

Let $\lambda$ be the smooth function on $M$ determined by $\Omega=\lambda \Omega_{a}$, where $\Omega_{a}$ is the area form of $M$, and let

$$
X_{\lambda}=X+\lambda V
$$

be the generating vector field of the magnetic flow $\phi\left(X_{\lambda}\right.$ also preserves Liouville measure).

From (5) we obtain:

$$
\left[V, X_{\lambda}\right]=H, \quad[V, H]=-X_{\lambda}+\lambda V, \quad\left[X_{\lambda}, H\right]=-\lambda X_{\lambda}+\left(K-H \lambda+\lambda^{2}\right) V .
$$

Note that

$$
H \lambda(x, v)=\langle\nabla \lambda(x), i v\rangle
$$

Lemma 3.1 (Pestov's identity). For every smooth function $\varphi: S M \rightarrow \mathbb{R}$ we have

$$
\begin{aligned}
2 H \varphi \cdot V X_{\lambda} \varphi & =\left(X_{\lambda} \varphi\right)^{2}+(H \varphi)^{2}-\left(K-H \lambda+\lambda^{2}\right)(V \varphi)^{2} \\
& +X_{\lambda}(H \varphi \cdot V \varphi)-H\left(X_{\lambda} \varphi \cdot V \varphi\right)+V\left(X_{\lambda} \varphi \cdot H \varphi\right) .
\end{aligned}
$$

Remark 3.2. A similar identity for the vector fields $X, H_{\lambda}:=H+\lambda V$ and $V$ was obtained in [16, Lemma 2.1]. 
Proof of Lemma 3.1. Using the commutation formulas, we deduce:

$$
\begin{aligned}
2 H \varphi \cdot V X_{\lambda} \varphi & -V\left(H \varphi \cdot X_{\lambda} \varphi\right) \\
& =H \varphi \cdot V X_{\lambda} \varphi-V H \varphi \cdot X_{\lambda} \varphi \\
& =H \varphi \cdot\left(X_{\lambda} V \varphi+\left[V, X_{\lambda}\right] \varphi\right)-X_{\lambda} \varphi \cdot(H V \varphi+[V, H] \varphi) \\
& =H \varphi \cdot\left(X_{\lambda} V \varphi+H \varphi\right)-X_{\lambda} \varphi \cdot\left(H V \varphi-X_{\lambda} \varphi+\lambda V \varphi\right) \\
& =\left(X_{\lambda} \varphi\right)^{2}+(H \varphi)^{2}+\left(X_{\lambda} V \varphi\right)(H \varphi)-(H V \varphi)\left(X_{\lambda} \varphi\right)-\lambda X_{\lambda} \varphi \cdot V \varphi \\
& =\left(X_{\lambda} \varphi\right)^{2}+(H \varphi)^{2}+X_{\lambda}(V \varphi \cdot H \varphi)-H\left(V \varphi \cdot X_{\lambda} \varphi\right)-\left[X_{\lambda}, H\right] \varphi \cdot V \varphi \\
& -\lambda X_{\lambda} \varphi \cdot V \varphi \\
& =\left(X_{\lambda} \varphi\right)^{2}+(H \varphi)^{2}+X_{\lambda}(V \varphi \cdot H \varphi)-H\left(V \varphi \cdot X_{\lambda} \varphi\right) \\
& -\left(K-H \lambda+\lambda^{2}\right)(V \varphi)^{2}
\end{aligned}
$$

which is equivalent to Pestov's identity.

Integrating Pestov's identity over $S M$ against the Liouville measure $d \mu$, we get

$$
\begin{aligned}
2 \int_{S M} H \varphi \cdot V X_{\lambda} \varphi d \mu & =\int_{S M}\left(X_{\lambda} \varphi\right)^{2} d \mu+\int_{S M}(H \varphi)^{2} d \mu \\
& -\int_{S M}\left(K-H \lambda+\lambda^{2}\right)(V \varphi)^{2} d \mu
\end{aligned}
$$

Let us derive one more integral identity. Let $\varphi$ be again an arbitrary smooth function on $S M$. By the commutation relations, we have

$$
X_{\lambda} V \varphi=V X_{\lambda} \varphi-H \varphi \text {. }
$$

Therefore,

$$
\left(X_{\lambda} V \varphi\right)^{2}=\left(V X_{\lambda} \varphi\right)^{2}+(H \varphi)^{2}-2 V X_{\lambda} \varphi \cdot H \varphi .
$$

Integrating, we obtain

$$
\int_{S M}\left(X_{\lambda} V \varphi\right)^{2} d \mu=\int_{S M}\left(V X_{\lambda} \varphi\right)^{2} d \mu+\int_{S M}(H \varphi)^{2} d \mu-2 \int_{S M} V X_{\lambda} \varphi \cdot H \varphi d \mu .
$$

Subtracting (6) from (7), we arrive at the final identity

$$
\begin{aligned}
\int_{S M}\left\{\left(X_{\lambda} V \varphi\right)^{2}-\left(K-H \lambda+\lambda^{2}\right)(V \varphi)^{2}\right\} & d \mu \\
& =\int_{S M}\left(V X_{\lambda} \varphi\right)^{2} d \mu-\int_{S M}\left(X_{\lambda} \varphi\right)^{2} d \mu .
\end{aligned}
$$

Let us now begin with the proof of Theorem B. If $X_{\lambda} \varphi=G(x)+\omega_{x}(v)$, then it is easy to see that the right-hand side of (8) is nonpositive. Indeed, since $\mu$ is invariant under $v \mapsto-v$ and $v \rightarrow i v$ we have

$$
\int_{S M} \omega_{x}(v) d \mu=0 \text { and } \int_{S M}\left(\omega_{x}(v)\right)^{2} d \mu=\int_{S M}\left(\omega_{x}(i v)\right)^{2} d \mu
$$


But $V X_{\lambda} \varphi=\omega_{x}(i v)$ and thus

$$
\int_{S M}\left(V X_{\lambda} \varphi\right)^{2} d \mu-\int_{S M}\left(X_{\lambda} \varphi\right)^{2} d \mu=-\int_{S M}(G(x))^{2} d \mu \leq 0 .
$$

Setting $\psi=V \varphi$, we get

$$
\int_{S M}\left\{\left(X_{\lambda} \psi\right)^{2}-\left(K-H \lambda+\lambda^{2}\right) \psi^{2}\right\} d \mu \leq 0 .
$$

We now show that this is possible if and only if $\psi=0$. This would give $V \varphi=0$, which says that $\varphi=f \circ \pi$ where $f$ is a smooth function on $M$. But in this case, since $d \pi_{(x, v)}\left(X_{\lambda}\right)=v$ we have $X_{\lambda}(\varphi)=d f_{x}(v)$. This clearly implies the claim of the theorem.

Lemma 3.3. If $\phi$ is Anosov, then for every closed magnetic geodesic $\gamma:[0, T] \rightarrow$ $M$ and every smooth function $z:[0, T] \rightarrow \mathbb{R}$ such that $z(0)=z(T)$ and $\dot{z}(0)=$ $\dot{z}(T)$ we have

$$
I:=\int_{0}^{T}\left\{\dot{z}^{2}-\left(K-\langle\nabla \lambda, i \dot{\gamma}\rangle+\lambda^{2}\right) z^{2}\right\} d t \geq 0
$$

with equality if and only if $z \equiv 0$.

Proof. Given $(x, v) \in S M$ and $\xi \in T_{(x, v)} T M$, let

$$
J_{\xi}(t)=d_{(x, v)}\left(\pi \circ \phi_{t}\right)(\xi) .
$$

We call $J_{\xi}$ a magnetic Jacobi field with initial condition $\xi$. It was shown in [14] that $J_{\xi}$ satisfies the following Jacobi equation:

$$
\ddot{J}_{\xi}+R\left(\dot{\gamma}, J_{\xi}\right) \dot{\gamma}-\left[Y\left(\dot{J}_{\xi}\right)+\left(\nabla_{J_{\xi}} Y\right)(\dot{\gamma})\right]=0,
$$

where $\gamma(t)=\pi \circ \phi_{t}(x, v), R$ is the curvature tensor of $g$ and $Y$ is determined by the equality $\Omega_{x}(u, v)=\left\langle Y_{x}(u), v\right\rangle$ for all $u, v \in T_{x} M$ and all $x \in M$. Let us express $J_{\xi}$ as follows:

$$
J_{\xi}(t)=x(t) \dot{\gamma}(t)+y(t) i \dot{\gamma}(t),
$$

and suppose in addition that $\xi \in T_{(x, v)} S M$, which implies

$$
g_{\gamma}\left(\dot{J}_{\xi}, \dot{\gamma}\right)=0
$$

A straightforward computation using (10) and (11) shows that $x$ and $y$ must satisfy the scalar equations:

$$
\begin{aligned}
& \dot{x}=\lambda(\gamma) y \\
& \ddot{y}+\left[K(\gamma)-\langle\nabla \lambda(\gamma), i \dot{\gamma}\rangle+\lambda^{2}(\gamma)\right] y=0 .
\end{aligned}
$$

Let $E$ be the weak stable bundle of $\phi$. Since for any $(x, v) \in S M$ the subspace $E$ does not intersect the vertical subspace $\operatorname{Ker} d \pi_{(x, v)}[13,10]$, there exists a linear map $S(x, v): T_{x} M \rightarrow T_{x} M$ such that $E$ can be identified with the graph of $S$. Let $u(x, v)$ be the trace of $S(x, v)$ and let $J_{\eta}=x \dot{\gamma}+y i \dot{\gamma}$ be the Jacobi field with 
initial conditions $\eta=(i v, S(i v)) \in E$. Since $u(t)=\langle S(t) i \dot{\gamma}, i \dot{\gamma}\rangle$ and $\dot{J}_{\eta}=S J_{\eta}$ we see that

$$
\dot{y}=u y
$$

Note that $y$ never vanishes. Given $z$ as in the hypothesis of the lemma, let $q$ be defined by the equation $z=q y$. Using equation (13) we have

$$
\begin{aligned}
I=-\int_{0}^{T} z\left(\ddot{z}+\left[K(\gamma)-\langle\nabla \lambda(\gamma), i \dot{\gamma}\rangle+\lambda^{2}(\gamma)\right] z\right) d t & =-\int_{0}^{T} q \frac{d}{d t}\left(\dot{q} y^{2}\right) d t \\
& =-\left[q \dot{q} y^{2}\right]_{0}^{T}+\int_{0}^{T} \dot{q}^{2} y^{2} d t
\end{aligned}
$$

Using the periodicity properties of $z$ and (14) we have

$$
\left[q \dot{q} y^{2}\right]_{0}^{T}=[z \dot{q} y]_{0}^{T}=-[q \dot{y} z]_{0}^{T}=-[q u y z]_{0}^{T}=-\left[u z^{2}\right]_{0}^{T}
$$

and the last term vanishes since $u$ is globally defined on $S M$. We conclude that

$$
I \geq 0
$$

with equality if and only $\dot{q} \equiv 0$. Hence if $I=0, q$ must be a constant, which must be zero since $y$ cannot be periodic in $T$.

We continue now with the proof of Theorem B. The last lemma, applied to the function $z=\psi(\gamma)$, yields

$$
\int_{\gamma}\left\{\left(X_{\lambda} \psi\right)^{2}-\left(K-H \lambda+\lambda^{2}\right) \psi^{2}\right\} d t \geq 0
$$

for every closed magnetic geodesic $\gamma$. Since the flow is Anosov, the invariant measures supported on closed orbits are dense in the space of all invariant measures on $S M$. Therefore, the above yields

$$
\int_{S M}\left\{\left(X_{\lambda} \psi\right)^{2}-\left(K-H \lambda+\lambda^{2}\right) \psi^{2}\right\} d \mu \geq 0 .
$$

Combining this with (8), we find that

$$
\int_{S M}\left\{\left(X_{\lambda} \psi\right)^{2}-\left(K-H \lambda+\lambda^{2}\right) \psi^{2}\right\} d \mu=0 .
$$

By the non-negative version of the Livšic theorem, proved independently by M. Pollicott and R. Sharp and by A. Lopes and P. Thieullen (see $[9,15]$ ), we conclude from (15) and (16) that

$$
\int_{\gamma}\left\{\left(X_{\lambda} \psi\right)^{2}-\left(K-H \lambda+\lambda^{2}\right) \psi^{2}\right\} d t=0
$$

for every closed magnetic geodesic $\gamma$. Applying again Lemma 3.3, we see that $\psi$ vanishes on all closed magnetic geodesics. Since the latter are dense in $S M$, the function $\psi$ vanishes on all of $S M$, as required. 


\section{Proof of Theorem C}

We begin with a general easy lemma. Given a smooth closed curve $\gamma:[0, T] \rightarrow$ $M$ and $k \in \mathbb{R}$ we define the free time action of $\gamma$ as:

$$
A_{k}(\gamma):=\frac{1}{2} \int_{0}^{T}|\dot{\gamma}(t)|^{2} d t+k T+c^{-1} \log \operatorname{hol}_{\alpha}(\gamma) \bmod 1 .
$$

Recall that the energy is the function given by $E(x, v):=\frac{1}{2}|v|_{x}^{2}$.

Lemma 4.1. Let $\gamma:[0, T] \rightarrow M$ be a closed magnetic geodesic with energy $k$. Let $\gamma_{\tau}:\left[0, T_{\tau}\right] \rightarrow M, \tau \in(-\varepsilon, \varepsilon)$, be a smooth variation of $\gamma$ by smooth closed curves. Then

$$
\frac{d A_{k}\left(\gamma_{\tau}\right)}{d \tau}(0)=0
$$

Proof. The curves $\gamma_{\tau}-\gamma$ form a 1-cycle which is the boundary of a 2-chain $\Sigma_{\tau}$. Then

$$
c^{-1} \log \operatorname{hol}_{\alpha}\left(\gamma_{\tau}\right)+c^{-1} \log \operatorname{hol}_{\alpha}(\gamma)=-\int_{\Sigma_{\tau}} \Omega \bmod 1 .
$$

If we let $W(t)$ be the variational vector field of $\gamma_{\tau}$, a straightforward calculation using that $\gamma$ has energy $k$ and (17) shows that

$$
\frac{d A_{k}}{d \tau}(0)=-\int_{0}^{T}\left\langle\frac{D \dot{\gamma}}{d t}, W(t)\right\rangle d t+\int_{0}^{T} \Omega(\dot{\gamma}(t), W(t)) d t
$$

Since $\gamma$ is a magnetic geodesic,

$$
\frac{D \dot{\gamma}}{d t}=Y_{\gamma}(\dot{\gamma})
$$

where $Y$ is determined by $\Omega_{x}(u, v)=\left\langle Y_{x}(u), v\right\rangle$. Thus

$$
\frac{d A_{k}}{d \tau}(0)=0
$$

Let us assume now that we are under the hypotheses of Theorem C.

Lemma 4.2. Suppose $\mathcal{S}_{\tau}=\mathcal{S}$ for all $\tau \in(-\varepsilon, \varepsilon)$. Then

$$
\int_{\gamma_{\tau}} \frac{d \beta_{\tau}}{d \tau}=0
$$

for every closed magnetic geodesic $\gamma_{\tau}$ of $\left(g, \Omega_{\tau}\right)$.

Proof. In each nontrivial homotopy class we have a 1-parameter family of closed magnetic geodesics $\gamma_{\tau}$. Let

$$
a_{\tau}\left(\gamma_{\tau}\right):=A_{1 / 2}^{\tau}\left(\gamma_{\tau}\right)=\ell(\gamma)+c^{-1} \log \operatorname{hol}_{\alpha_{\tau}}(\gamma) \bmod 1 .
$$

Since $\mathcal{S}$ is countable and the map $(-\varepsilon, \varepsilon) \ni \tau \mapsto a_{\tau}\left(\gamma_{\tau}\right)$ is continuous we have

$$
a_{\tau}\left(\gamma_{\tau}\right)=a_{\tau_{0}}\left(\gamma_{\tau_{0}}\right)
$$


for all $\tau \in(-\varepsilon, \varepsilon)$. Since

$$
\log \operatorname{hol}_{\alpha_{\tau}}(\sigma)=\log \operatorname{hol}_{\alpha_{\tau_{0}}}(\sigma)+\frac{1}{2 \pi} \int_{\sigma}\left(\beta_{\tau}-\beta_{\tau_{0}}\right) \bmod 1
$$

for all $\sigma$, we have:

$$
a_{\tau}\left(\gamma_{\tau}\right)=a_{\tau_{0}}\left(\gamma_{\tau}\right)+\frac{1}{2 \pi c} \int_{\gamma_{\tau}}\left(\beta_{\tau}-\beta_{\tau_{0}}\right) \bmod 1 .
$$

By Lemma 4.1, the map $\tau \mapsto a_{\tau_{0}}\left(\gamma_{\tau}\right)$ has a critical point at $\tau=\tau_{0}$, hence the last equality implies

$$
\left.\frac{d}{d \tau}\right|_{\tau=\tau_{0}} \int_{\gamma_{\tau}}\left(\beta_{\tau}-\beta_{\tau_{0}}\right)=0
$$

which is easily seen to imply

$$
\left.\int_{\gamma_{\tau_{0}}} \frac{d \beta_{\tau}}{d \tau}\right|_{\tau=\tau_{0}}=0
$$

To complete the proof of Theorem C, observe that the previous lemma and Theorem B imply that for each $\tau, \frac{d \beta_{\tau}}{d \tau}$ is exact. If we let $f_{\tau}$ be a primitive of $\frac{d \beta_{\tau}}{d \tau}$, then

$$
F_{\tau}:=\int_{0}^{\tau} f_{s} d s
$$

are the required functions.

Remark 4.3. The proofs of Theorem $\mathrm{C}$ and its corollary work in any dimension provided that Theorem B holds in any dimension. One only needs the cohomology class $[\Omega]$ to be rational, i.e. there exists $\lambda \in \mathbb{R}$ such that $[\lambda \Omega]$ is an integral class.

Even if $[\Omega]$ is not rational, we can still attach to the magnetic flow an action spectrum by considering a torus bundle $\mathbb{T}^{r}$ over $M$. The action spectrum is now a subset of $\mathbb{T}^{r}$ and the same infinitesimal rigidity holds, provided that the magnetic flow is Anosov.

The question of whether Theorem B extends to higher dimension is more delicate. We hope to discuss these topics elsewhere, as well as the analogue of Theorem B for higher order tensors.

\section{References}

[1] K. Burns, G.P. Paternain, Anosov magnetic flows, critical values and topological entropy, Nonlinearity 15 (2002) 281-314.

[2] C.B. Croke, V.A. Sharafutdinov, Spectral rigidity of a negatively curved manifold, Topology 37 (1998) 1265-1273.

[3] N.S. Dairbekov, V.A. Sharafutdinov, Some problems of integral geometry on Anosov manifolds, Ergodic Theory Dynam. Systems 23 (2003) 59-74.

[4] P. Foulon, B. Hasselblatt, Zygmund strong foliations, Israel J. Math. 138 (2003) 157-169. 
[5] V. Guillemin, D. Kazhdan, Some inverse spectral results for negatively curved 2manifolds, Topology 19 (1980) 301-312.

[6] V. Guillemin, A. Uribe, Circular symmetry and trace formula, Invent. Math. 96 (1989) $385-423$.

[7] S. Hurder, A. Katok, Differentiability, rigidity and Godbillon-Vey classes for Anosov flows, Publ. Math. IHES 72 (1990) 5-61.

[8] R. de la Llave, J.M. Marco, R. Moriyon, Canonical perturbation theory of Anosov systems and regularity for the Livsic cohomology equation, Ann. Math. 123 (1986) 537-611.

[9] A.O. Lopes and P. Thieullen, Sub-actions for Anosov flows, Ergodic Theory Dynam. Systems 25 (2005) 605-628.

[10] G.P. Paternain, On Anosov energy levels of Hamiltonians on twisted cotangent bundles, Bulletin of the Brazilian Math. Society Vol 252 (1994) 207-211.

[11] On the regularity of the Anosov splitting for twisted geodesic flows, Math. Res. Lett. 4 (1997) 871-888.

[12] _ The longitudinal KAM-cocycle of a magnetic flow, to appear in Math. Proc. Cambridge Philos. Soc.

[13] G. P. Paternain, M. Paternain, On Anosov Energy Levels of Convex Hamiltonian Systems, Math. Z. 217 (1994) 367-376.

[14] _ Anosov geodesic flows and twisted symplectic structures, International Congress on Dynamical Systems in Montevideo (a tribute to Ricardo Mañé), F Ledrappier, J. Lewowicz, S. Newhouse eds, Pitman Research Notes in Math. 362 (1996), 132-145.

[15] M. Pollicott, R. Sharp, Livsic theorems, maximising measures and the stable norm, Dynamical Systems: An International Journal 19 (2004) 75-88.

[16] V.A. Sharafutdinov, G. Uhlmann, On deformation boundary rigidity and spectral rigidity of Riemannian surfaces with no focal points, J. Diff. Geom. 56 (2000) 93-110.

Kazakh British Technical University, Tole Bi 59, 050000 Almaty, Kazakhstan

E-mail address: nurlan.dairbekov@gmail.com

Department of Pure Mathematics and Mathematical Statistics, University of Cambridge, Cambridge CB3 0WB, England

E-mail address: g.p.paternain@dpmms.cam.ac.uk 